\title{
NLO Monte Carlo predictions for heavy-quark production at the LHC: pp collisions in ALICE
}

\author{
M. Klasen, ${ }^{a}$ C. Klein-Bösing, ${ }^{b, c}$ K. Kovarik, ${ }^{a}$ G. Kramer, ${ }^{d}$ M. Topp ${ }^{a}$ and J.P. Wessels ${ }^{b}$ \\ ${ }^{a}$ Institut für Theoretische Physik, Westfälische Wilhelms-Universität Münster, \\ Wilhelm-Klemm-Straße 9, D-48149 Münster, Germany \\ ${ }^{b}$ Institut für Kernphysik, Westfälische Wilhelms-Universität Münster, \\ Wilhelm-Klemm-Straße 9, D-48149 Münster, Germany \\ ${ }^{c}$ ExtreMe Matter Institute, GSI, \\ Planckstraße 1, D-64291 Darmstadt, Germany \\ ${ }^{d}$ II. Institut für Theoretische Physik, Universität Hamburg, \\ Luruper Chaussee 149, D-22761 Hamburg, Germany \\ E-mail: michael.klasen@uni-muenster.de, \\ c.klein-boesing@uni-muenster.de, karol.kovarik@uni-muenster.de, \\ kramer@mail.desy.de, m_topp02@uni-muenster.de, \\ j.wessels@uni-muenster.de
}

ABSTRACT: Next-to-leading order (NLO) QCD predictions for the production of heavy quarks in proton-proton collisions are presented within three different approaches to quark mass, resummation and fragmentation effects. In particular, new NLO and parton shower simulations with POWHEG are performed in the ALICE kinematic regime at three different centre-of-mass energies, including scale and parton density variations, in order to establish a reliable baseline for future detailed studies of heavy-quark suppression in heavyion collisions. Very good agreement of POWHEG is found with FONLL, in particular for centrally produced $D^{0}, D^{+}$and $D^{*+}$ mesons and electrons from charm and bottom quark decays, but also with the generally somewhat higher GM-VFNS predictions within the theoretical uncertainties. The latter are dominated by scale rather than quark mass variations. Parton density uncertainties for charm and bottom quark production are computed here with POWHEG for the first time and shown to be dominant in the forward regime, e.g. for muons coming from heavy-flavour decays. The fragmentation into $D_{s}^{+}$mesons seems to require further tuning within the NLO Monte Carlo approach.

Keywords: Monte Carlo Simulations, Hadronic Colliders

ARXIV EPRINT: 1405.3083 


\section{Contents}

1 Introduction 1

2 Theoretical input $\quad 2$

2.1 FONLL 3

2.2 GM-VFNS 5

2.3 POWHEG 6

$\begin{array}{lll}3 & \text { LHC predictions } & \mathbf{7}\end{array}$

3.1 pp collisions at $\sqrt{s}=2.76 \mathrm{TeV} \quad 8$

$\begin{array}{ll}3.2 \text { pp collisions at } \sqrt{s}=7 \mathrm{TeV} & 10\end{array}$

$\begin{array}{ll}3.3 \text { pp collisions at } \sqrt{s}=5.02 \mathrm{TeV} & 14\end{array}$

4 Conclusions $\quad 15$

\section{Introduction}

The production of heavy quarks in hadronic collisions is an important probe for various aspects of QCD. In proton-proton collisions, this process is perturbatively calculable down to low transverse momenta $\left(p_{T}\right)$ due to the presence of the heavy-quark mass $m$. Calculations can there be performed reliably in the Fixed Flavour Number Scheme (FFNS), where the heavy flavour appears only in the hard scattering process. Conversely, when $p_{T} \gg m$ the Zero-Mass Variable Flavour Number Scheme (ZM-VFNS) applies, where the heavy quark appears also as an active parton in the initial-state Parton Density Functions (PDFs) and final-state Fragmentation Functions (FFs) and its mass can be neglected in the hard scattering process. The intermediate regime requires delicate matching procedures, that have been developed under the names of Fixed Order plus Next-to-Leading Logarithms (FONLL) [1] and General-Mass Variable Flavour Number Scheme (GM-VFNS) [2].

While these calculations are mostly analytic and provide only an accurate description of the inclusively produced heavy hadron, the matching of next-to-leading order (NLO) calculations with parton showers with the MC@NLO [3] or POWHEG [4] methods allows to provide a more complete description of the hadronic final state. It is modeled by parton showers and string or cluster fragmentation and can be subject to decays and even detector response, while at the same time NLO QCD accuracy in the hard and improved leadinglogarithm resummation in the soft/collinear regimes are retained. In this respect these predictions are therefore superior to those from general-purpose Monte Carlo generators like PYTHIA [5] or HERWIG [6], which also provide a complete hadronic final state, but only with leading order accuracy. 
In this paper we compare the three different theoretical approaches mentioned above in the kinematic regime relevant for the ALICE experiment at the LHC. The goal is to establish the reliability of the proton-proton baseline for future studies of heavy-ion collisions, where the suppression (quenching) of heavy quarks at large transverse momenta is an important signal for the deconfined state of matter, the so-called quark-gluon plasma (QGP). To this end, we compute, with FONLL, GM-VFNS and POWHEG, the production of $D^{0}$, $D^{+}, D^{*+}$, and $D_{s}^{+}$mesons in pp collisions at $\sqrt{s}=2.76$ and $7 \mathrm{TeV}$ as well as the production of muons and electrons originating from heavy-flavour (charm and bottom quark) decays at these energies. For the latter, we also make predictions at a centre-of-mass energy of $\sqrt{s}=$ $5.02 \mathrm{TeV}$, pertinent to the $2013 \mathrm{pPb}$ run of the LHC whose data are currently being analysed by the experimental collaborations. We also assess the theoretical uncertainties coming from independent variations of the renormalisation and factorisation scales, the heavyquark mass, and from the complete error set of CTEQ6.6 parton densities [7]. Factorisation scheme uncertainties can in principle also be important [8], but are usually not considerered.

Previously, only the FONLL and GM-VFNS calculations had been compared with each other for $D^{0}, D^{+}$and $D^{*+}$ meson production at $\sqrt{s}=2.76 \mathrm{TeV}[9]$ and $7 \mathrm{TeV}$ [10] and for $D_{s}^{+}$meson production also at $7 \mathrm{TeV}[11]$. On the other hand, MC@NLO had been tested against NLO in the FFNS [3] and POWHEG against MC@NLO [4] for top and bottom quark production at the Tevatron and at the nominal LHC energy of $\sqrt{s}=14 \mathrm{TeV}$. More recently, FONLL, MC@NLO and POWHEG predictions have been compared for $D^{+}$and $B^{+}$meson production at an energy of $7 \mathrm{TeV}$ [12], but no predictions were made for other charm mesons or at the centre-of-mass energies of 2.76 and $5.02 \mathrm{TeV}$. Furthermore, a systematic scale and PDF uncertainty study has only been performed there for FONLL and not for POWHEG, and no comparisons to the GM-VFNS have been made. Attempts to describe the quenching of heavy quarks are mostly and at least in principle based on QCD, but they range in practice from the consideration of radiative energy loss supplemented with in-medium dissociation [13] or collisional energy loss [14-17] over a Boltzmann approach to multi-parton scatterings [18] to the relativistic Langevin equation with multiple uncorrelated random collisions [19, 20] and AdS/CFT drag coefficients [21]. Note, however, that the description of heavy-quark quenching is beyond the scope of our present work.

This paper is organised as follows: in section 2 we describe the relevant features of the three different theoretical approaches (FONLL, GM-VFNS and POWHEG) that we employ and compare in this work. Section 3 contains our main numerical results, i.e. central predictions and associated theoretical uncertainties for transverse-momentum distributions of heavy quarks produced in the ALICE kinematic regime at the LHC with three different centre-of-mass energies. We summarise our results and present our conclusions in section 4.

\section{Theoretical input}

In this section, we describe the main features of the three different theoretical approaches to heavy-quark production (FONLL, GM-VFNS and POWHEG), that will later form the bases of our numerical predictions for pp collisions at various centre-of-mass energies of the LHC. The most important parameter is of course the heavy-quark mass $m$, which we take 
to be $1.5 \mathrm{GeV}$ for charm and $4.75 \mathrm{GeV}$ for bottom quarks. These are the default values in both FONLL and POWHEG and have in particular been used to obtain the central FONLL predictions in the ALICE publications [9-11, 22-25]. In the GM-VFNS calculation the quark mass dependence is of course much less pronounced than in the massive calculations.

For the same reasons and in line with common practice, we employ as our default central renormalisation and factorisation scales the square root of the quadratic mean of the heavy-quark mass $m$ and the transverse momentum $p_{T}$ multiplied by $\sqrt{2}$ and set $\mu=$ $\mu_{f}=\sqrt{m^{2}+p_{T}^{2}}$. This scale is also used as the default value of the final-state fragmentation scale $\mu_{D}$, which in GM-VFNS predictions can be varied independently of the initial-state factorisation scale $\mu_{f}$. Independent variations of these scales will be performed by factors of two about the central value, but omitting the extreme combinations that amount to factors of four between the different scales.

As our central PDF set we employ the CTEQ6.6 parametrisation [7], again because it was already used in the FONLL calculations shown in the ALICE publications. Note, however, that these PDFs have been obtained with $m_{c}=1.3 \mathrm{GeV}$ and $m_{b}=4.5 \mathrm{GeV}$, which are slightly lower than our default quark mass values. This influences in particular the $c$ and $b$ quark PDFs, which enter the evolution equations with zero input at the starting scales $\mu_{0}=m_{c}$ and $m_{b}$. The value of the QCD scale in CTEQ6.6 in the $\overline{\mathrm{MS}}$ scheme and for five light quark flavors is $\Lambda_{\overline{\mathrm{MS}}}^{n_{f}=5}=226 \mathrm{MeV}$, which gives $\alpha_{s}\left(M_{Z}\right)=0.1181$ at $M_{Z}=91.1876 \mathrm{GeV}$ in agreement with the world average of $\alpha_{s}\left(M_{Z}\right)=0.1184 \pm 0.0007$ [26]. The PDF uncertainty will be estimated in POWHEG using the usual formulas

$$
\begin{aligned}
& \delta^{+} f=\sqrt{\sum_{i=1}^{22}\left[\max \left(f_{i}^{(+)}-f_{0}, f_{i}^{(-)}-f_{0}, 0\right)\right]^{2}}, \\
& \delta^{-} f=\sqrt{\sum_{i=1}^{22}\left[\max \left(f_{0}-f_{i}^{(+)}, f_{0}-f_{i}^{(-)}, 0\right)\right]^{2}},
\end{aligned}
$$

where $f_{i}^{ \pm}$are the PDFs for positive and negative variations of the PDF parameters along the $i$-th eigenvector direction in the 22-dimensional PDF parameter space.

In the following, we turn to the description of the specific theoretical assumputions entering the FONLL, GM-VFNS and POWHEG approaches.

\section{$2.1 \quad$ FONLL}

The Fixed-Order plus Next-to-Leading-Logarithms (FONLL) calculation of Cacciari et al. [1] is based on the matching of NLO massive and massless calculations according to the prescription

$$
\mathrm{d} \sigma_{\mathrm{FONLL}}=\mathrm{d} \sigma_{\mathrm{FO}}+\left(\mathrm{d} \sigma_{\mathrm{RS}}-\mathrm{d} \sigma_{\mathrm{FOMO} 0}\right) \times G\left(m, p_{T}\right) .
$$

Here, FO denotes the massive NLO cross section, where the heavy-quark flavour $h$ enters only in the partonic scattering through so-called flavour creation processes, but not in the PDFs, and its mass is kept as a non-vanishing parameter. The NLO partonic cross section 
then includes mass logarithms of the form

$$
\mathrm{d} \sigma_{\mathrm{FO}} \propto \frac{\alpha_{s} C_{F}}{2 \pi}\left(\frac{1+z^{2}}{1-z}\right)_{+} \log \frac{p_{T}^{2}}{m^{2}}+\ldots
$$

where $m$ regularises the collinear singularity, e.g. of the splitting $h \rightarrow h g$, and where the logarithm becomes very large when $p_{T} \gg m$, thus spoiling the perturbative expansion in $\alpha_{s}$. This part, which is singular in the massless limit $(m \rightarrow 0)$, and the finite parts related to its different definition in dimensional and mass regularisation are denoted FOM0 and therefore resummed to NLL order in the contribution denoted RS. RS is then added to the FO calculation, while the overlap FOM0 is subtracted to avoid double counting.

The resummation relies on the perturbative FFs for the probability of a heavy quark $h$, gluon, or light parton $i$ to go into a heavy quark $h$ [27], which satisfy Altarelli-Parisi evolution equations. Their initial values at the starting scale $\mu_{0}$ are calculable perturbatively. In the $\overline{\mathrm{MS}}$ scheme they are given by

$$
\begin{aligned}
& D_{h}\left(z, \mu_{0}\right)=\delta(1-z)+\frac{\alpha_{s}\left(\mu_{0}\right) C_{F}}{2 \pi}\left[\frac{1+z^{2}}{1-z}\left(\log \frac{\mu_{0}^{2}}{m^{2}}+2 \log (1-z)-1\right)\right]_{+}, \\
& D_{g}\left(z, \mu_{0}\right)=\frac{\alpha_{s}\left(\mu_{0}\right) T_{F}}{2 \pi}\left(z^{2}+(1-z)^{2}\right) \log \frac{\mu_{0}^{2}}{m^{2}}, \text { and } \\
& D_{i}\left(z, \mu_{0}\right)=0, \quad \text { for } i \neq g, h,
\end{aligned}
$$

respectively. The second term relates to the gluon-splitting contribution $g \rightarrow h \bar{h}$, while the third term describes the coupling of light to heavy quarks. It occurs only at next-to-nextto-leading order through $i \rightarrow i g \rightarrow i h \bar{h}$ and is therefore neglected. As usual, $C_{F}=4 / 3$ and $T_{F}=1 / 2$.

The perturbative FFs are evolved to the factorisation scale $\mu_{f}$ and convoluted with the NLO cross sections for massless partons, subtracted in the $\overline{\mathrm{MS}}$ scheme, so that also socalled flavour excitation processes are included. These involve the heavy quark also as an active parton in the PDFs. The result is then convoluted with non-perturbative functions to describe the hadronisation of heavy quarks into heavy hadrons. For bottom quarks, the functional form

$$
D_{b}(z)=(\alpha+1)(\alpha+2) z^{\alpha}(1-z)
$$

is used, which is normalised to unity and where the single parameter $\alpha=24.2$ has been fitted to LEP data relevant to the production of a mixture of $B$ hadrons, since no data are available for individual hadrons like $B^{0}$ or $B^{+}$[28]. For charm quarks, experimental data for individual mesons $\left(D^{0}, D^{+}, D^{*}\right.$, and $\left.D_{s}^{+}\right)$exist. They have been exploited, together with the theoretical understanding of the similarities and differences in the fragmentation of a heavy quark into pseudoscalar $(D)$ and vector $\left(D^{*}\right)$ mesons, to construct non-perturbative functions for $D$ mesons exclusively in terms of $c \rightarrow D^{*}$ fragmentation. Its single parameter $r=0.1$, relating $D$ to $D^{*}$ mesons, was extracted from LEP data, together with the different branching ratios, and also used to describe primary pseudoscalar $D$ meson production [29]. The fragmentation is numerically performed by rescaling the quark three-momentum at a constant angle in the laboratory frame. The decay of the $D$ and $B$ mesons into electrons 
(or muons) is controlled by the experimentally measured decay spectra using data from the BaBar and CLEO collaborations [30], normalised to branching ratios at high energy [26].

The matching function $G\left(m, p_{T}\right)$ must tend to unity in the massless limit $p_{T} \gg m$, where FO approaches FOM0 and the mass logarithms must be resummed. However, in FONLL its functional form is not simply unity, but rather

$$
G\left(m, p_{T}\right)=\frac{p_{T}^{2}}{p_{T}^{2}+a^{2} m^{2}}
$$

While it fulfils the above condition, the matching is not exact away from the massless limit. This is justified with the observation that the difference RS - FOM0, although formally of next-to-next-to-leading order, turns out to be abnormally large below $p_{T}=5 \mathrm{~m}$, so that the constant $a$ is phenomenologically set to $a=5$. Indeed, the FOM0 and RS results exhibit different approximately logarithmic mass dependences, that are of higher order in $\alpha_{s}$ as expected, but that can amount to $50 \%$ for physical values of the strong coupling constant and values of $p_{T} \sim m$ [1]. Suppressing the difference RS - FOM0 in this regime with the above matching function and $a=5$ reduces it artificially to the level of two percent at the price of introducing arbitrariness and associated theoretical uncertainty. Furthermore, the FOM0 and RS results are evaluated by replacing the transverse momentum $p_{T}$ with the transverse mass $m_{T}=\sqrt{p_{T}^{2}+m^{2}}$, so that all contributions are evaluated at the same $m_{T}$. The central values of the renormalisation and factorisation scale are also identified in all parts with $m_{T}$.

Predictions for $c$ and $b$ quark production at the LHC with a centre-of-mass energy of $\sqrt{s}=7 \mathrm{TeV}$ have been presented in ref. [12], where also the charm and bottom quark masses have been varied from $1.3-1.7 \mathrm{GeV}$ and $4.5-5.0 \mathrm{GeV}$, respectively. Note that FONLL predictions can only be made for inclusive heavy-quark distributions. In particular, it is not possible to study correlations of the produced heavy quark or hadron with other objects in the final state. The produced heavy hadron is of course always collinear to the heavy quark, and there is also no information on the kinematical distribution of its decay products.

\subsection{GM-VFNS}

The same restrictions also apply to the GM-VFNS calculation, which has also been performed for inclusive distributions of heavy hadrons. It was originally performed in the massless limit, valid at high $p_{T}$, and therefore includes flavour creation, gluon splitting and flavour excitation processes. Subsequently the calculation was improved by identifiying the previously omitted finite mass terms through a comparison with the massive NLO calculation, where together with the mass logarithms also finite terms were subtracted in such a way that in the limit $m \rightarrow 0$ the correct massless $\overline{\mathrm{MS}}$ result was recovered [31]. This is necessary, since the PDFs and FFs that are convoluted with the partonic cross sections are defined in the ZM-VFNS. Heavy-quark mass terms in flavour excitation processes were neglected, which corresponds to a specific choice of scheme (known as S-ACOT), but no loss in precision [32].

Massless non-perturbative $D$-meson FFs were obtained in ref. [33] from Belle, CLEO, ALEPH and OPAL data using a starting scale of $\mu_{0}=m_{c}=1.5 \mathrm{GeV}$ for all partons up to the charm quark and $m_{b}=5.0 \mathrm{GeV}$ for bottom quarks. Although the value of $m_{b}$ is slightly 
higher here than our default value of $4.75 \mathrm{GeV}$, the effect on charm meson production is not expected to be very large. In the theoretical NLO calculation, finite-mass corrections were included in the production cross sections, i.e. logarithmic singularities were subtracted together with finite terms, so that the correct massless limit was recovered when $m_{q} \rightarrow 0$. The optimal functional ansatz at the starting scale for $c$ and $b$ quarks fragmenting into charmed mesons turned out to be [34]

$$
D_{h}\left(z, \mu_{0}\right)=N z^{-\left(1+\gamma^{2}\right)}(1-z)^{a} e^{-\gamma^{2} / z}
$$

with three free parameters, which were fitted separately to $D^{0}, D^{+}$and $D^{*+}$ data. GMFVNS predictions for $D$-meson production have previously been compared to ALICE data obtained in pp collisions at the LHC with a centre-of-mass energy of $7 \mathrm{TeV}[10,35]$. In the predictions for $D_{s}$ mesons [35], somewhat older FFs functions [36] had to be used.

Massless non-perturbative $B$-meson FFs were obtained in ref. [37] from ALEPH, OPAL and SLD data using a starting scale of $\mu_{0}=m_{b}=4.5 \mathrm{GeV}$ (i.e. slightly lower than our default value of $4.75 \mathrm{GeV}$ ) for the initial bottom quark FF. All other FFs vanished there. Again, in the theoretical NLO calculation finite-mass corrections were included in the production cross sections, but not in the FFs. A standard functional ansatz at the starting scale

$$
D_{h}\left(z, \mu_{0}\right)=N z^{\alpha}(1-z)^{\beta}
$$

was found to describe the experimental data very well. In agreement with previous findings using the FONLL approach [30] it was found that using the Peterson form at the starting scale does not lead to a good description of the data. Since only inclusive $B$-meson data are available, separate fits for $B^{0}$ and $B^{+}$mesons were not possible. GM-VNFS predictions have been compared to inclusive $B$-meson data from CMS obtained in pp collisions at $7 \mathrm{TeV}$ centre-of-mass energy [38] and also to ALICE, ATLAS and CMS data on leptonic decays of charm and bottom production at 2.76 and $7 \mathrm{TeV}$ centre-of-mass energy [39]. Furthermore, predictions have been made for $D$ mesons produced at $7 \mathrm{TeV}$ in $B$ decays using either a two-step transition $b \rightarrow B \rightarrow D$, based on $b \rightarrow B$ FFs and $B \rightarrow D$ spectra as measured by CLEO, or a one-step transition based on FFs for $b \rightarrow D$ [40]. Note that in the GM-VFNS approach the theoretical uncertainty is estimated varying three independent scales $\left(\mu, \mu_{f}\right.$, and $\mu_{D}$ ), not only two as in FONLL (where $\mu_{f}=\mu_{D}$ ), and no fixed phenomenomenological matching function $G\left(m, p_{T}\right)$ is used, which generally leads to larger uncertainty bands, in particular at low $p_{T}$.

\subsection{POWHEG}

In contrast to the FONLL and GM-VFNS approaches, which are based on NLO calculations and the factorisation of heavy-quark FFs and are thus limited to the description of the inclusive production of heavy quarks and mesons, general purpose Monte Carlo generators based on parton showers and string or cluster fragmentation allow for a more complete description of the final state, including decay kinematics, particle identification and, if needed, even detector response. Their precision has now been enhanced to NLO in the hard and improved leading-logarithm accuracy in the soft/collinear regime through 
a consistent combination of NLO calculations with parton showers, made possible by the proper subtraction of doubly counted contributions in the soft and collinear regions.

For heavy-quark production, this was first achieved in MC@NLO [3] and subsequently in POWHEG [4]. Like MC@NLO, the POWHEG approach is based on a massive NLO calculation, i.e. the massive quarks are not active partons in the PDFs and large logarithms are not resummed into heavy-quark PDFs. Heavy flavours can, however, be excited through the initial-state parton shower or be produced in gluon splittings. For a correct matching of parton showers and PDFs, that is performed as in standard (leading order) Monte Carlo programs, five-flavour strong coupling constant and PDFs are used in MC@NLO and POWHEG, the terms $-\alpha_{s} \frac{2 T_{F}}{3 \pi} \log \frac{\mu^{2}}{m^{2}} \sigma_{q \bar{q}}^{(0)}$ and $-\alpha_{s} \frac{2 T_{F}}{3 \pi} \log \frac{\mu^{2}}{\mu_{f}^{2}} \sigma_{g g}^{(0)}$ are added to the $q \bar{q}$ and $g g$ channel cross sections, and the heavy flavour is ignored in the PDFs. The error committed in this way is of higher order in $\alpha_{s}$ and numerically small. Due to the complex colour flow in parton-parton scattering, the Sudakov form factor for light partons in POWHEG has currently only leading-logarithmic accuracy. I.e., in contrast to FONLL, only a subset of the large logarithms are resummed. However, at small and moderate $p_{T}$ the NLO and parton shower approach should be superior to FONLL, since it has almost the same accuracy in this region, but in addition allows for a complete and fully exclusive description of final state. This approach should eventually also permit to include rescattering effects in the medium in heavy-ion collisions.

In this work, the heavy-quark part of POWHEG BOX 2.1 has been called for each centre-of-mass energy from a self-written C++ code together with LHAPDF 5.8.9 [41] and PYTHIA 8.175 [5] to generate ten million events for both charm and bottom quark pairs, then to shower, hadronise and decay them to stable particles. Note that the hadronisation, performed in PYTHIA using the Lund string model, has been tuned to data at leading, not next-to-leading order accuracy. The event files were then analysed for heavy $D$ and $B$ mesons and their electron and muon decay products. Kinematic (in particular rapidity) cuts were applied, and binned $p_{T}$ differential cross sections, normalised to the total NLO POWHEG cross section, were calculated for every set of PDFs and choice of scale combination. The total PDF error was obtained according to eqs. (2.1) and (2.2) and stored separately from the largest scale error.

\section{LHC predictions}

Let us now turn to our numerical predictions for heavy-quark production in pp collisions at the LHC with centre-of-mass energies of $\sqrt{s}=2.76,5.02$ and $7 \mathrm{TeV}$ in the ALICE kinematic regime. In the following figures, the statistical and systematic uncertainties of the ALICE data will always be shown as in the experimental publications as black error bars and boxes, respectively. The theoretical predictions will appear as shaded bands, which in the case of FONLL (green bands) comprise variations of renormalisation scale $\mu$ and common factorisation scale $\mu_{f}$ as well as variations of the heavy quark mass, added in quadrature. In the case of GM-VFNS (black dashed lines), they comprise the maximal error (yellow bands) due to variations of the renormalisation scale $\mu$, PDF factorisation scale $\mu_{f}$ and FF factorisation scale $\mu_{D}$, and in the case of POWHEG (red full lines) they comprise on the one hand 
the maximal error due to variations of the renormalisation scale $\mu$ and PDF factorisation scale $\mu_{f}$ (light blue bands) and on the other hand the error due to the PDF uncertainty as described above (dark blue bands). For future comparisons of theoretical predictions with nuclear collision data it is important to not only know the central prediction with CTEQ6.6 PDFs (red), but also with CTEQ6.1 PDFs [42] (not shown), which is used as the basis of nuclear PDFs, in particular EPS09 [43]. The set CTEQ6.1 has been obtained with the same heavy-quark masses and value of $\Lambda_{\overline{\mathrm{MS}}}^{n_{f}=5}$ as CTEQ6.6. We have verified that the two central predictions coincide well in the kinematic regimes considered here.

\section{1 pp collisions at $\sqrt{s}=2.76 \mathrm{TeV}$}

At $\sqrt{s}=2.76 \mathrm{TeV}$, the ALICE collaboration has measured the $p_{T}$ distributions of $D^{0}, D^{+}$ and $D^{*+}$ mesons produced centrally with rapidity $|y|<0.5$ from $p_{T}=1 \mathrm{GeV}$ (for $D^{0}$ ) and $2 \mathrm{GeV}$ (for $D^{+}$and $D^{*+}$ ) to $12 \mathrm{GeV}$ [9]. These data are shown in figure 1. Within their respective uncertainties, which can amount to almost an order of magnitude at low $p_{T}$, all three theoretical calculations agree with the data. The two massive FONLL and POWHEG calculations show a tendency to underestimate the data, in particular at low $p_{T}$, whereas the originally massless GM-VFNS calculation shows the opposite tendency and tends to slightly overestimate the data, but agrees very well with them already at intermediate to larger $p_{T}$ values. This corresponds well with the expectation that in this regime, quark mass effects should no longer play an important role. As expected from previous studies, the scale uncertainties dominate over quark mass and PDF uncertainties, so that the FONLL, GM-VFNS and POWHEG theoretical error bands are all comparable in size despite their different decompositions. In particular, the PDF uncertainty, which was computed here with POWHEG for the first time, amounts to half or less of the scale uncertainty in the whole kinematic range, reflecting the good knowledge of PDFs in the intermediate range of $x$ or $x_{T}=2 p_{T} / \sqrt{s}=0.001-0.02$ relevant here.

The ALICE collaboration has also analysed the production of $D^{0}, D^{+}$and $D^{*+}$ mesons in $\mathrm{PbPb}$ collisions with $\sqrt{s_{N N}}=2.76 \mathrm{TeV}$ [44]. They compared them to scaled pp data from $\sqrt{s}=7 \mathrm{TeV}$ on the basis of the theoretical energy dependence, as the above data set was not yet available at the time, and established a nuclear modification factor $R_{A A}$ of about 0.3 for central and 0.6 for peripheral heavy-ion collisions. In this work, we are only concerned with pp collisions and do not attempt to describe nuclear suppression effects.

The ALICE collaboration has furthermore measured muons from heavy-flavour decay at forward rapidity of $2.5<y<4$ in both pp and $\mathrm{PbPb}$ collisions [22] and observed similar nuclear suppression effects as in prompt ( $B$ feed-down subtracted) charmed meson production. In this inclusive muon measurement, contributions from charm and bottom quarks were not separated, but the main backgrounds from pion and kaon decays were removed. In figure 2 the ALICE pp data are compared with the three theoretical predictions in the range $2 \mathrm{GeV}<p_{T}<10 \mathrm{GeV}$. We find again generally good agreement and a tendency of the originally massive calculations to slightly underestimate the data. The central GM-VFNS predictions agree with the data perfectly well (see also figure 3 in the Erratum of ref. [39]). In the POWHEG prediction, the PDF uncertainty, computed here for the first time, has become the dominating source of uncertainty in this forward regime and in particular at 

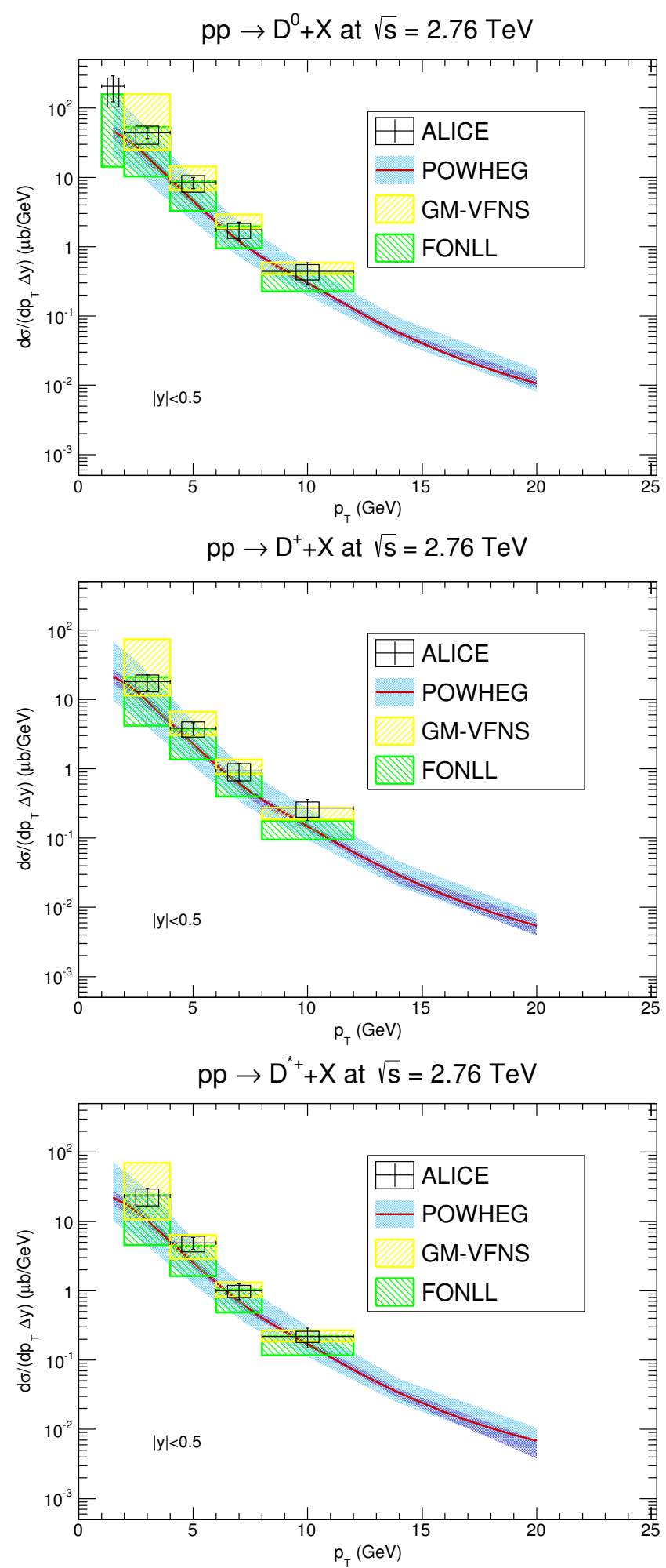

Figure 1. Transverse-momentum distributions of $D^{0}$ (top), $D^{+}$(centre) and $D^{*+}$ (bottom) mesons centrally produced at the LHC with $\sqrt{s}=2.76 \mathrm{TeV}$ and compared to ALICE data [9]. 


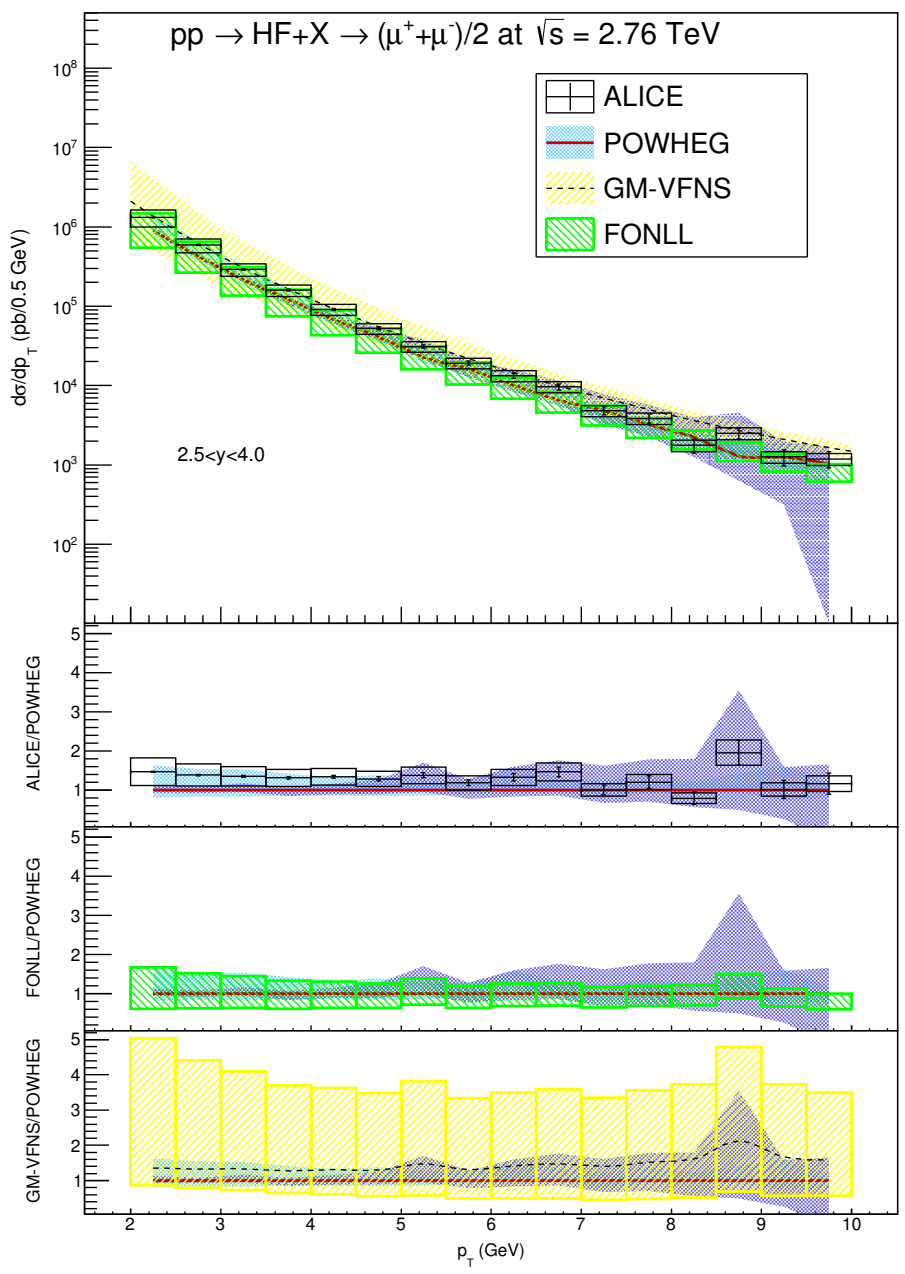

Figure 2. Transverse-momentum distributions of muons from heavy-flavour (charm and bottom quark) decay produced in the forward region at the LHC with $\sqrt{s}=2.76 \mathrm{TeV}$ and compared to ALICE data [22].

large $p_{T}$. This reflects the fact that we are now in an asymmetric situation, probing $x$ values down to $10^{-4}$ in one proton and above 0.1 in the other. In both regions the PDFs are known with much less precision than at intermediate values of $x$. Statistical Monte Carlo fluctuations can sometimes even lead to very large deviations, in particular at large $p_{T}$. As one can see from the ratio plots in figure 2, the differences of ALICE data, FONLL and GM-VFNS vs. POWHEG concern mostly the normalisation and not the shape of the distributions.

\section{2 pp collisions at $\sqrt{s}=7 \mathrm{TeV}$}

The prompt production of $D^{0}, D^{+}$and $D^{*+}$ mesons has been analysed by the ALICE collaboration also at $\sqrt{s}=7 \mathrm{TeV}[10]$. As at the lower centre-of-mass energy, the charmed mesons were identified through their decays $D^{0} \rightarrow K^{-} \pi^{+}, D^{+} \rightarrow K^{-} \pi^{+} \pi^{+}, D^{*+} \rightarrow D^{0} \pi^{+}$and their charge conjugates. Feed-down from $B$-meson decays was subtracted using FONLL. 
The corresponding $p_{T}$-distributions at central rapidity $|y|<0.5$ are shown in figure 3 . The discussion from the previous section applies here again in the sense that within the considerable theoretical uncertainties the data can be described in all three approaches. The main difference is that the data extend now out to $p_{T}$ values of $16 \mathrm{GeV}$ for $D^{0}$ and $24 \mathrm{GeV}$ for $D^{+}$ and $D^{*+}$. At large $p_{T}$, where mass effects become less important, the GM-VFNS prediction shows the largest stability, followed by FONLL, which resums mass effects into a perturbative FF at next-to-leading logarithmic level, whereas in POWHEG these logarithms are only partially resummed through the Sudakov factor in the parton shower. At low $p_{T}$, only the FONLL and POWHEG predictions are shown to agree with the data, since the GM-VFNS central prediction and its scale uncertainty rise there rapidly and in particular do not show the specific turnover of the data as the genuinely massive calculations [35]. ${ }^{1}$ As before, the PDF uncertainties remain subdominant over the whole kinematic range.

For centrally produced $D_{s}^{+}$mesons, which were reconstructed by ALICE through the decay $D_{s}^{+} \rightarrow \phi \pi^{+}$, with $\phi \rightarrow K^{-} K^{+}$, and its charged conjugate [11], the $p_{T^{-} \text {-spectrum }}$ extends only from 2 to $12 \mathrm{GeV}$, as can be seen in figure 4 . The GM-VFNS predictions agree here very well with the data, whereas POWHEG falls short of them even when taking into account its uncertainty band. This may be seen as an indication that the mass of the strange quark leads to a further suppression of the predicted rate and/or that the fragmentation into bound states of charm and strange quarks is less well described by the Lund string model than the one into heavy-light mesons and may thus require more tuning to data. As the ratio plots in figure 4 show, the ALICE data and GM-VFNS predictions differ from POWHEG mostly in normalisation, but also somewhat in shape.

Similarly to the measurements at $\sqrt{s}=2.76 \mathrm{TeV}$, ALICE has measured muons produced from heavy-flavour decay without flavour separation and after subtraction of the backgrounds from pion and kaon decays in the forward region $2.5<y<4$ [25]. These data are shown in figure 5 together with the three different theoretical predictions in the range $2 \mathrm{GeV}<p_{T}<12 \mathrm{GeV}$. The central GM-VFNS prediction lies somewhat higher than at the lower centre-of-mass energy in particular at high $p_{T}$ (see also figure 3 in the Erratum of ref. [39]), but within the considerable scale uncertainty the GM-VFNS predictions still agree with the ALICE data, as do the two other predictions from FONLL and POWHEG. The PDF uncertainties, computed only with POWHEG, exceed those from the scale variations at intermediate and high $p_{T}$, where again very small and large values of $x$ are probed, respectively. The ALICE data, FONLL and GM-VFNS predictions have in addition been divided into five equidistant rapidity bins and successfully compared there, but we refrain here from showing the corresponding figures and POWHEG predictions as they do not add significant information.

At central rapidities $(|y|<0.5)$, ALICE has furthermore measured heavy-flavour decay into electrons without flavour separation [23]. The main backgrounds here stem from pseudoscalar, light and heavy vector meson decays, which have been subtracted, together with real and virtual photon conversions, using a Monte Carlo "cocktail" calculation [23].

\footnotetext{
${ }^{1}$ We take the opportunity to correct the misprints in the figure captions of ref. [35], where the centre-ofmass energy should read $\sqrt{s}=7 \mathrm{TeV}$, not $\mathrm{GeV}$. It has been shown that for a specific combination of scales, the GM-VFNS predictions can be brought into agreement with the data also at low $p_{T}[45]$.
} 

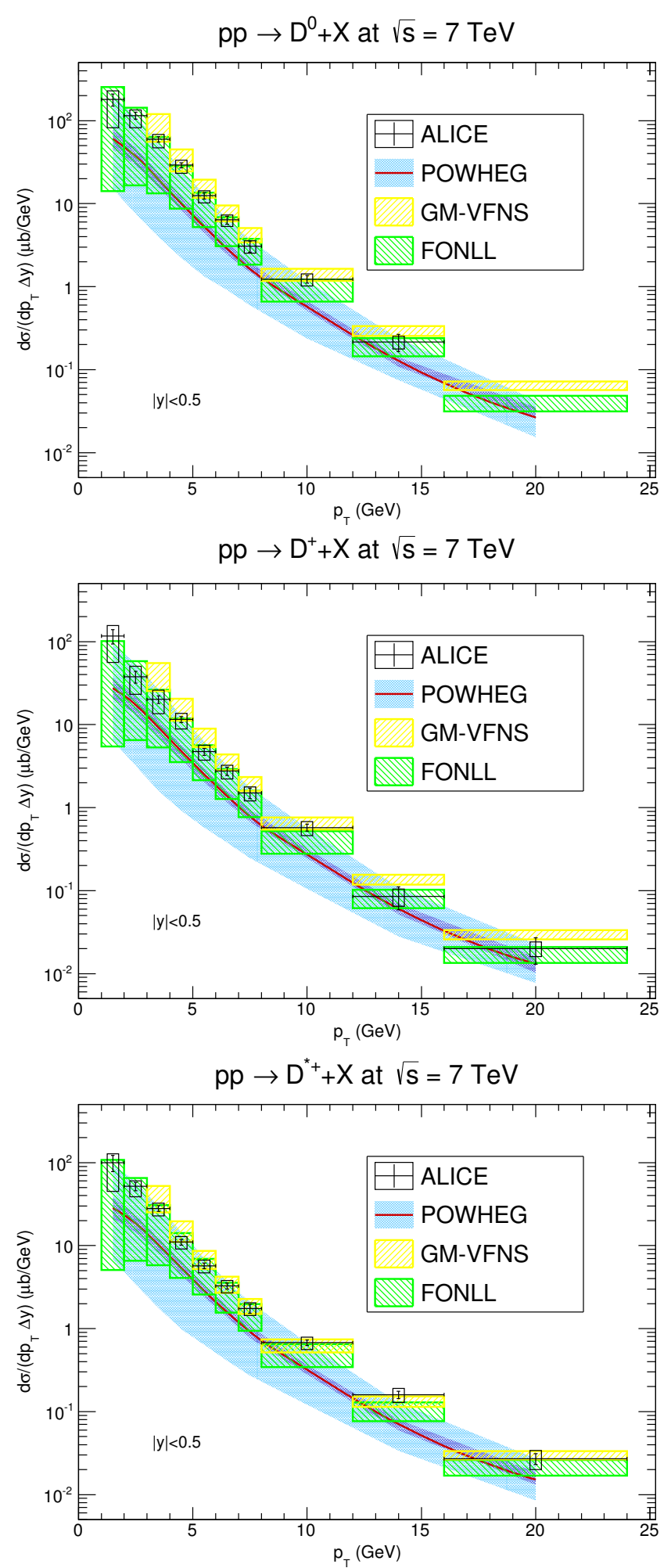

Figure 3. Transverse-momentum distributions of $D^{0}$ (top), $D^{+}$(centre) and $D^{*+}$ (bottom) mesons centrally produced at the LHC with $\sqrt{s}=7 \mathrm{TeV}$ and compared to ALICE data [10]. 


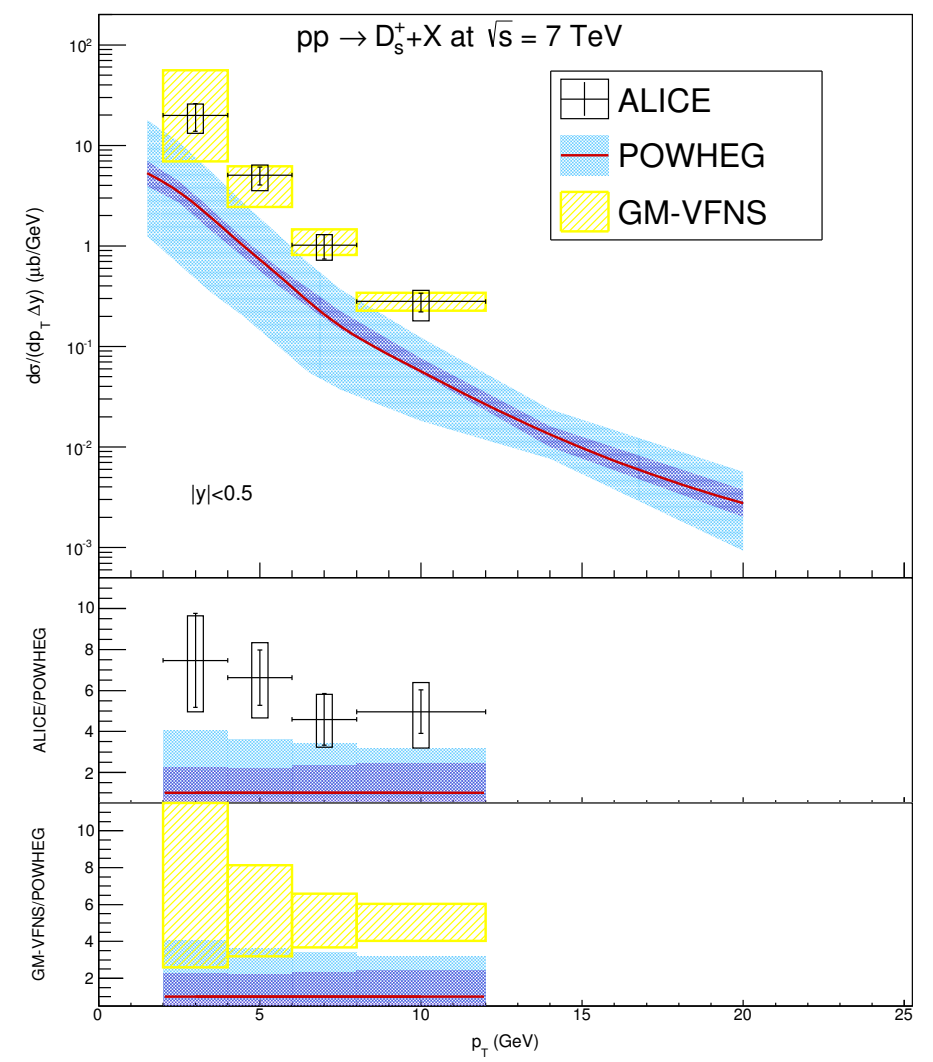

Figure 4. Transverse-momentum distributions of $D_{s}^{+}$mesons centrally produced at the LHC with $\sqrt{s}=7 \mathrm{TeV}$ and compared to ALICE data [11].

A comparison with FONLL predictions is included in the experimental publication, while a comparison with GM-VFNS predictions can be found in figure 3 in the Erratum of ref. [39]. The measurement was subsequently repeated including flavour separation, where decays of beauty hadrons were identified through a secondary vertex, displaced from the primary collision vertex [24]. For this data set, comparisons with FONLL have been made in the experimental publication and with GM-VFNS in ref. [39], but only for the decays of bottom hadrons. As one can see in figure 6 (bottom), the theoretical uncertainty for the latter is very large at small $p_{T}$, whereas it is much smaller for charm decays, as can also be seen in figure 6 (top) and as it should be for smaller quark masses. For beauty decays, the POWHEG prediction and its theoretical uncertainty coincide almost exactly with the FONLL predictions over the entire $p_{T}$ range, the PDF uncertainty being again subdominant in this central kinematic regime. For charm decays, only the central POWHEG prediction and its upper uncertainty band limit coincide with FONLL, the lower edge being somewhat lower. In this case, the PDF uncertainty becomes visible and comparable to, albeit still smaller than the scale error at larger $p_{T}$. The excellent agreement among FONLL and POWHEG is indeed quite remarkable and much better for inclusive leptons than for inclusive mesons, which obviously depend much more on the fragmentation model than the decay leptons. 


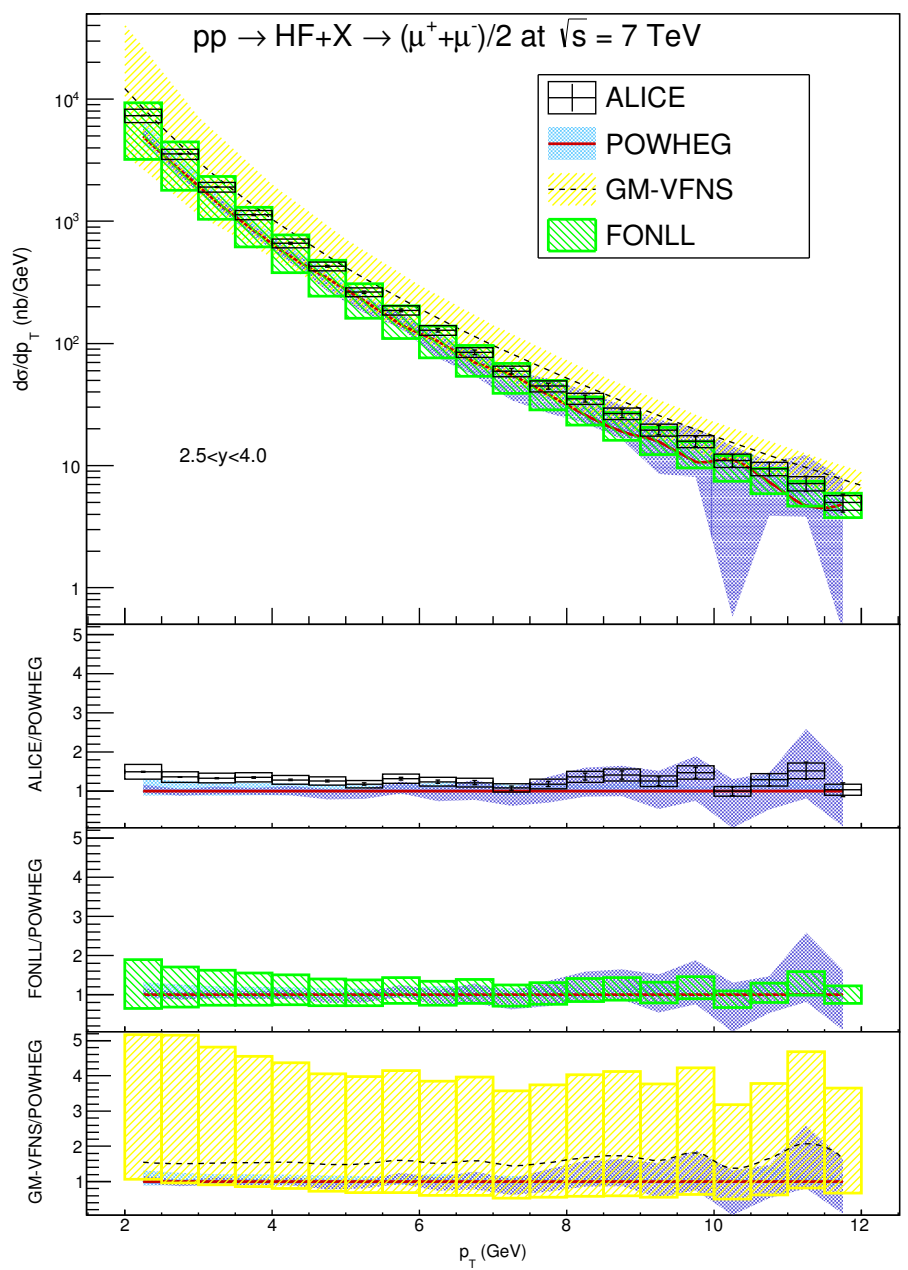

Figure 5. Transverse-momentum distributions of muons from heavy-flavour (charm and bottom quark) decay produced in the forward region at the LHC with $\sqrt{s}=7 \mathrm{TeV}$ and compared to ALICE data [25].

\section{3 pp collisions at $\sqrt{s}=5.02 \mathrm{TeV}$}

Finally, we turn to pp collisions at a centre-of-mass energy of $\sqrt{s}=5.02 \mathrm{TeV}$, relevant also for $\mathrm{pPb}$ collisions, where no reference calculations are published yet. In figure 7 we show new predictions for centrally produced electrons from heavy-flavour decays with POWHEG and GM-VFNS in the kinematic regime that is currently under analysis by the ALICE collaboration, i.e. for transverse momenta from $1 \mathrm{GeV}$ to $8 \mathrm{GeV}$. As before, the general trend of good agreement within scale uncertainty bands, subdominant PDF uncertainties and a tendency of GM-VFNS to lie above POWHEG, in particular at low $p_{T}$, continues here. As before, the GM-VFNS scale uncertainty at low $p_{T}$ is larger for beauty than for charm hadrons due to the larger bottom quark mass. It will be interesting to learn if the ALICE data in $\mathrm{pPb}$ also agree with both theoretical predictions as was the case for $\mathrm{pp}$ reactions at higher and lower energies. 

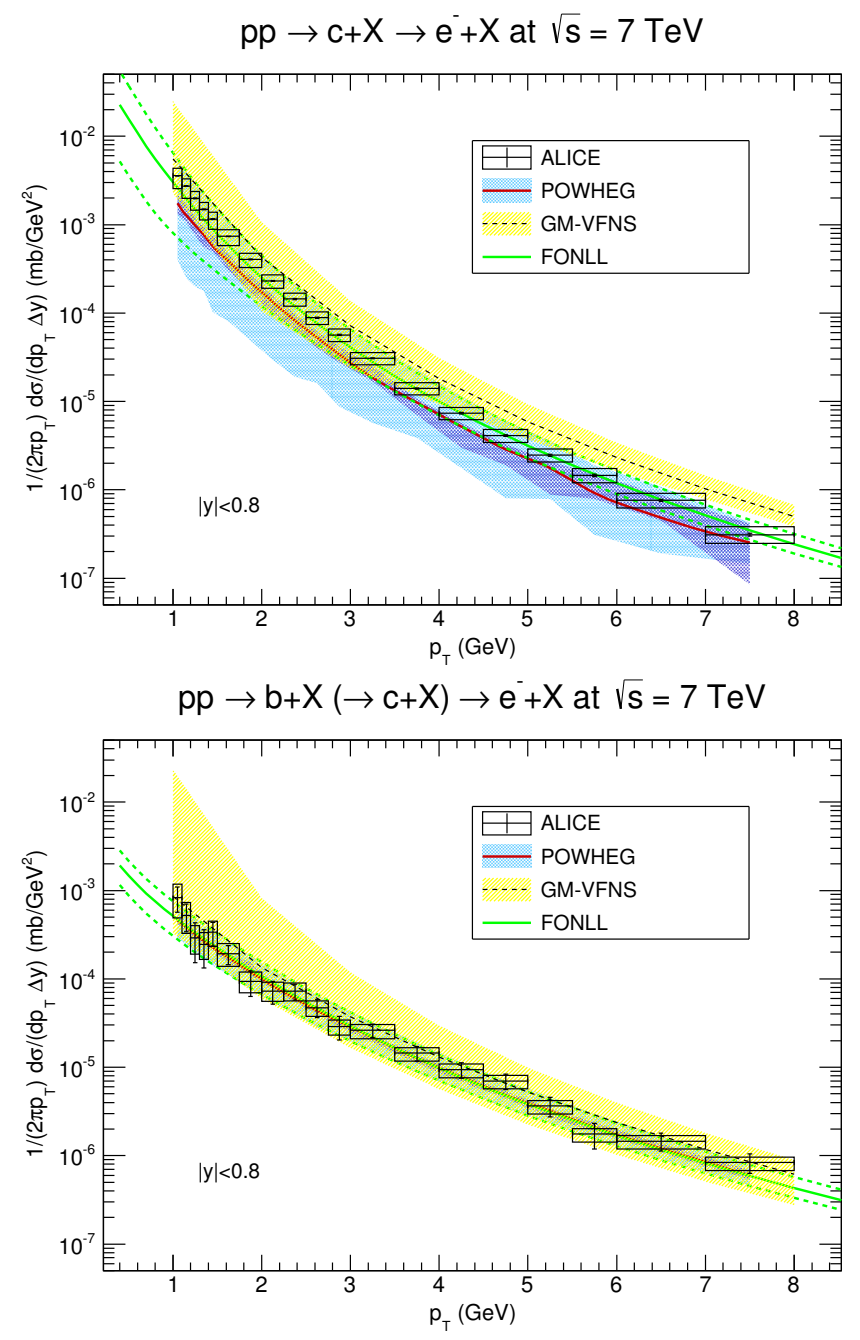

Figure 6. Transverse-momentum distributions of electrons from charm (top) and bottom (bottom) quark decay centrally produced at the LHC with $\sqrt{s}=7 \mathrm{TeV}$ and compared to ALICE data [24].

\section{Conclusions}

In this paper, motivated by the importance of a solid theoretical understanding of the pp baseline for future analyses of heavy-ion collision data, we have presented an extensive theoretical analysis of heavy-quark production in the ALICE kinematic regime at the LHC. In addition to the originally massive FONLL and massless GM-VFNS calculations, which were partially already available, but which only allow for limited comparisons of selected inclusive meson or decay lepton distributions, we have performed detailed calculations with the NLO Monte Carlo program POWHEG. These allow in principle for a more exclusive description of the final state, including e.g. correlations of the heavy quarks with other particles, and eventually also for the modelling of medium modifications.

In line with our goal of establishing a pp baseline, we have concentrated here on detailed comparisons of the FONLL, GM-VFNS and POWHEG approaches to inclusive transversemomentum spectra of heavy mesons or decay leptons, at central and forward rapidities, and 

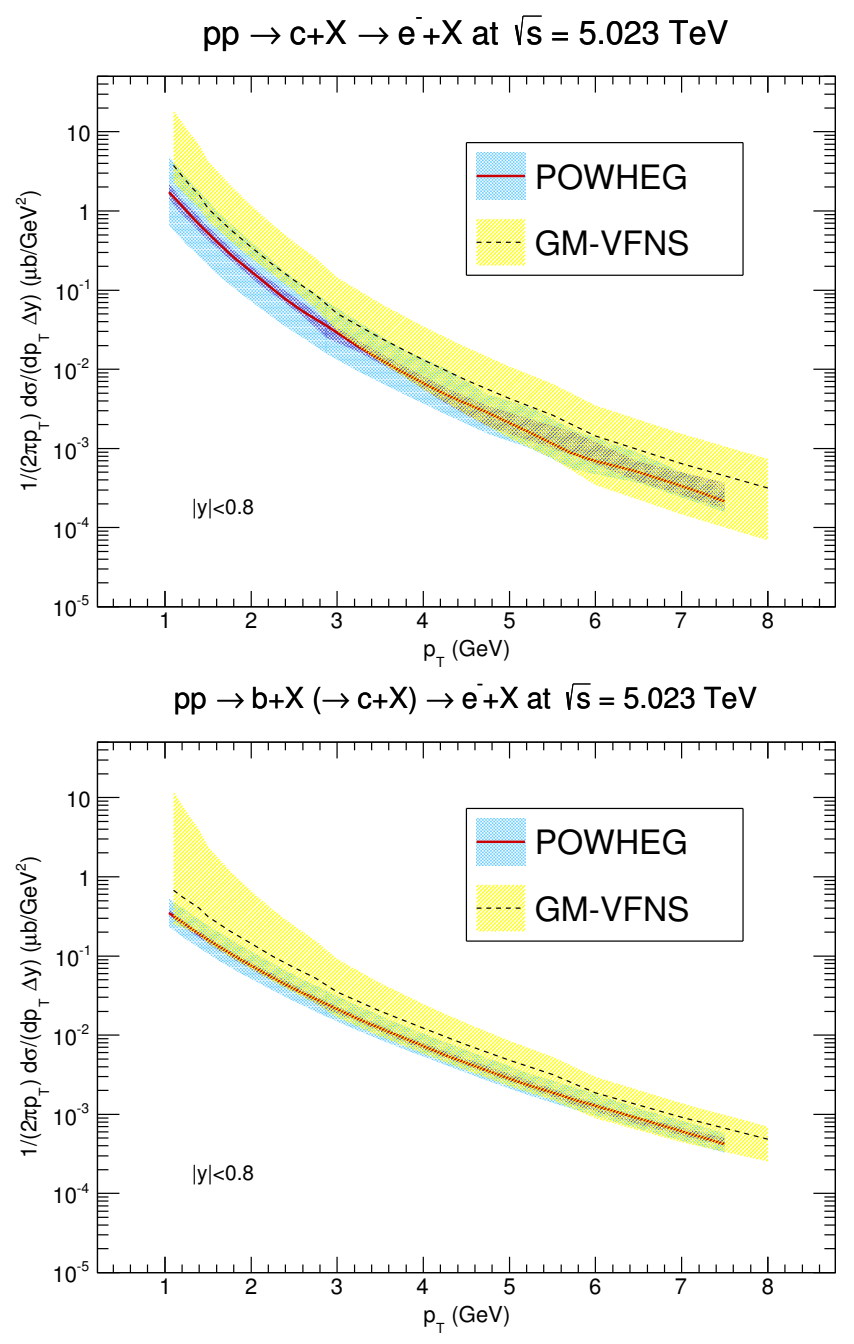

Figure 7. Transverse-momentum distributions of electrons from charm (top) and bottom (bottom) quark decay centrally produced at the LHC with $\sqrt{s}=5.02 \mathrm{TeV}$, pertinent to $\mathrm{pPb}$ collisions during 2013.

at three different centre-of-mass energies. Within the respective theoretical uncertainties, which were defined slightly differently in the three cases, but which were always dominated by scale variations, we found good agreement among the three theoretical calculations. For centrally produced electrons, the agreement among FONLL and POWHEG turned out to be indeed quite remarkable, while the hadronisation model affected more the inclusive meson spectra, in particular for $D_{s}^{+}$mesons.

PDF uncertainties were analysed for the first time and obtained here with the POWHEG approach with the result that they become dominant only in the forward regime and/or at large transverse momenta, corresponding to asymmetric situations and the regimes of very small and very large $x$. There, the PDFs are still known with insufficient precision and could be determined better using heavy-quark pp data, but e.g. also data from vector boson production [46, 47]. A better knowledge of proton and nuclear PDFs will in particular be important to distinguish cold from hot nuclear effects and to understand central vs. peripheral collisions and individual vs. collective phenomena. 
Clearly this work is only a first step towards more detailed theoretical investigations, which will include further differential distributions, two-particle correlations, protonnucleus and nucleus-nucleus collisions, and ultimately collective phenomena. Our extensive NLO Monte Carlo data sample produced with POWHEG provides a solid basis for such studies, where often only the analysis routines must be adapted, whereas the generated data can be used for multiple purposes.

\section{Acknowledgments}

We thank M. Heide and H. Spiesberger for making several previously obtained FONLL and GM-VFNS results available to us. The work of C. Klein-Bösing was supported by the Helmholtz Alliance Program of the Helmholtz Association, contract HA216/EMMI "Extremes of Density and Temperature: Cosmic Matter in the Laboratory".

Open Access. This article is distributed under the terms of the Creative Commons Attribution License (CC-BY 4.0), which permits any use, distribution and reproduction in any medium, provided the original author(s) and source are credited.

\section{References}

[1] M. Cacciari, M. Greco and P. Nason, The $p_{T}$ spectrum in heavy flavor hadroproduction, JHEP 05 (1998) 007 [hep-ph/9803400] [INSPIRE].

[2] B.A. Kniehl, G. Kramer, I. Schienbein and H. Spiesberger, Inclusive $D^{* \pm}$ production in $p \bar{p}$ collisions with massive charm quarks, Phys. Rev. D 71 (2005) 014018 [hep-ph/0410289] [INSPIRE].

[3] S. Frixione, P. Nason and B.R. Webber, Matching NLO QCD and parton showers in heavy flavor production, JHEP 08 (2003) 007 [hep-ph/0305252] [INSPIRE].

[4] S. Frixione, P. Nason and G. Ridolfi, A positive-weight next-to-leading-order Monte Carlo for heavy flavour hadroproduction, JHEP 09 (2007) 126 [arXiv:0707.3088] [INSPIRE].

[5] T. Sjöstrand, S. Mrenna and P.Z. Skands, A brief introduction to PYTHIA 8.1, Comput. Phys. Commun. 178 (2008) 852 [arXiv:0710.3820] [INSPIRE].

[6] G. Corcella et al., HERWIG 6: an event generator for hadron emission reactions with interfering gluons (including supersymmetric processes), JHEP 01 (2001) 010 [hep-ph/0011363] [INSPIRE].

[7] P.M. Nadolsky et al., Implications of CTEQ global analysis for collider observables, Phys. Rev. D 78 (2008) 013004 [arXiv: 0802.0007] [inSPIRE].

[8] M. Klasen and G. Kramer, Large transverse momentum jet production and DIS distributions of the proton, Phys. Lett. B 386 (1996) 384 [hep-ph/9605210] [InSPIRE].

[9] ALICE collaboration, Measurement of charm production at central rapidity in proton-proton collisions at $\sqrt{s}=2.76 \mathrm{TeV}$, JHEP 07 (2012) 191 [arXiv:1205.4007] [INSPIRE].

[10] ALICE collaboration, Measurement of charm production at central rapidity in proton-proton collisions at $\sqrt{s}=7 \mathrm{TeV}$, JHEP 01 (2012) 128 [arXiv:1111.1553] [INSPIRE].

[11] ALICE collaboration, $D_{s}^{+}$meson production at central rapidity in proton-proton collisions at $\sqrt{s}=7$ TeV, Phys. Lett. B 718 (2012) 279 [arXiv:1208.1948] [INSPIRE]. 
[12] M. Cacciari et al., Theoretical predictions for charm and bottom production at the LHC, JHEP 10 (2012) 137 [arXiv: 1205.6344] [INSPIRE].

[13] Y. He, I. Vitev and B.-W. Zhang, $\mathcal{O}\left(\alpha_{s}^{3}\right)$ analysis of inclusive jet and di-jet production in heavy ion reactions at the Large Hadron Collider, Phys. Lett. B 713 (2012) 224 [arXiv:1105.2566] [INSPIRE].

[14] W.A. Horowitz and M. Gyulassy, Quenching and tomography from RHIC to LHC, J. Phys. G 38 (2011) 124114 [arXiv:1107.2136] [InSPIRE].

[15] A. Buzzatti and M. Gyulassy, Jet flavor tomography of quark gluon plasmas at RHIC and LHC, Phys. Rev. Lett. 108 (2012) 022301 [arXiv:1106.3061] [INSPIRE].

[16] P.B. Gossiaux, R. Bierkandt and J. Aichelin, Tomography of a quark gluon plasma at RHIC and LHC energies, Phys. Rev. C 79 (2009) 044906 [arXiv:0901.0946] [INSPIRE].

[17] P.B. Gossiaux, J. Aichelin, T. Gousset and V. Guiho, Competition of heavy quark radiative and collisional energy loss in deconfined matter, J. Phys. G 37 (2010) 094019 [arXiv: 1001.4166] [INSPIRE].

[18] O. Fochler, J. Uphoff, Z. Xu and C. Greiner, Jet quenching and elliptic flow at RHIC and LHC within a pQCD-based partonic transport model, J. Phys. G 38 (2011) 124152 [arXiv: 1107.0130] [INSPIRE].

[19] W.M. Alberico et al., Heavy-flavour spectra in high energy nucleus-nucleus collisions, Eur. Phys. J. C 71 (2011) 1666 [arXiv:1101.6008] [INSPIRE].

[20] M. Monteno et al., Heavy-flavor dynamics in nucleus-nucleus collisions: from RHIC to LHC, J. Phys. G 38 (2011) 124144 [arXiv:1107.0256] [INSPIRE].

[21] W.A. Horowitz, Testing $p Q C D$ and AdS/CFT energy loss at RHIC and LHC, AIP Conf. Proc. 1441 (2012) 889 [arXiv:1108.5876] [INSPIRE].

[22] ALICE collaboration, Production of muons from heavy flavour decays at forward rapidity in pp and $\mathrm{Pb}-\mathrm{Pb}$ collisions at $\sqrt{s_{\mathrm{NN}}}=2.76 \mathrm{TeV}$, Phys. Rev. Lett. 109 (2012) 112301 [arXiv: 1205.6443] [INSPIRE].

[23] ALICE collaboration, Measurement of electrons from semileptonic heavy-flavour hadron decays in pp collisions at $\sqrt{s}=7$ TeV, Phys. Rev. D 86 (2012) 112007 [arXiv:1205.5423] [INSPIRE].

[24] ALICE collaboration, Measurement of electrons from beauty hadron decays in pp collisions at $\sqrt{s}=7$ TeV, Phys. Lett. B 721 (2013) 13 [arXiv:1208.1902] [INSPIRE].

[25] ALICE collaboration, Heavy flavour decay muon production at forward rapidity in proton-proton collisions at $\sqrt{s}=7$ TeV, Phys. Lett. B 708 (2012) 265 [arXiv:1201.3791] [INSPIRE].

[26] Particle Data Group collaboration, J. Beringer et al., Review of particle physics (RPP), Phys. Rev. D 86 (2012) 010001 [inSPIRE].

[27] B. Mele and P. Nason, The fragmentation function for heavy quarks in QCD, Nucl. Phys. B 361 (1991) 626 [INSPIRE].

[28] M. Cacciari, P. Nason and C. Oleari, A study of heavy flavored meson fragmentation functions in $e^{+} e^{-}$annihilation, JHEP 04 (2006) 006 [hep-ph/0510032] [INSPIRE].

[29] M. Cacciari and P. Nason, Charm cross-sections for the Tevatron run II, JHEP 09 (2003) 006 [hep-ph/0306212] [INSPIRE]. 
[30] M. Cacciari, P. Nason and R. Vogt, QCD predictions for charm and bottom production at RHIC, Phys. Rev. Lett. 95 (2005) 122001 [hep-ph/0502203] [INSPIRE].

[31] B.A. Kniehl, G. Kramer, I. Schienbein and H. Spiesberger, Collinear subtractions in hadroproduction of heavy quarks, Eur. Phys. J. C 41 (2005) 199 [hep-ph/0502194] [INSPIRE].

[32] . Kramer, Michael, F.I. Olness and D.E. Soper, Treatment of heavy quarks in deeply inelastic scattering, Phys. Rev. D 62 (2000) 096007 [hep-ph/0003035] [INSPIRE].

[33] T. Kneesch, B.A. Kniehl, G. Kramer and I. Schienbein, Charmed-meson fragmentation functions with finite-mass corrections, Nucl. Phys. B 799 (2008) 34 [arXiv:0712.0481] [INSPIRE].

[34] M.G. Bowler, $e^{+} e^{-}$production of heavy quarks in the string model, Z. Phys. C 11 (1981) 169 [inSPIRE].

[35] B.A. Kniehl, G. Kramer, I. Schienbein and H. Spiesberger, Inclusive charmed-meson production at the CERN LHC, Eur. Phys. J. C 72 (2012) 2082 [arXiv:1202.0439] [INSPIRE].

[36] B.A. Kniehl and G. Kramer, Charmed-hadron fragmentation functions from CERN LEP1 revisited, Phys. Rev. D 74 (2006) 037502 [hep-ph/0607306] [INSPIRE].

[37] B.A. Kniehl, G. Kramer, I. Schienbein and H. Spiesberger, Finite-mass effects on inclusive B meson hadroproduction, Phys. Rev. D 77 (2008) 014011 [arXiv:0705.4392] [INSPIRE].

[38] B.A. Kniehl, G. Kramer, I. Schienbein and H. Spiesberger, Inclusive B-meson production at the LHC in the GM-VFN scheme, Phys. Rev. D 84 (2011) 094026 [arXiv:1109.2472] [INSPIRE].

[39] P. Bolzoni and G. Kramer, Inclusive lepton production from heavy-hadron decay in pp collisions at the LHC, Nucl. Phys. B 872 (2013) 253 [Erratum ibid. B 876 (2013) 334] [arXiv: 1212.4356] [INSPIRE].

[40] P. Bolzoni and G. Kramer, Inclusive charmed-meson production from bottom hadron decays at the LHC, J. Phys. G 41 (2014) 075006 [arXiv:1310.2924] [InSPIRE].

[41] M.R. Whalley, D. Bourilkov and R.C. Group, The Les Houches accord PDFs (LHAPDF) and LHAGLUE, hep-ph/0508110 [INSPIRE].

[42] D. Stump et al., Inclusive jet production, parton distributions and the search for new physics, JHEP 10 (2003) 046 [hep-ph/0303013] [INSPIRE].

[43] K.J. Eskola, H. Paukkunen and C.A. Salgado, EPS09: a new generation of NLO and LO nuclear parton distribution functions, JHEP 04 (2009) 065 [arXiv:0902.4154] [INSPIRE].

[44] ALICE collaboration, Suppression of high transverse momentum D mesons in central $\mathrm{Pb}-\mathrm{Pb}$ collisions at $\sqrt{s_{\mathrm{NN}}}=2.76 \mathrm{TeV}$, JHEP 09 (2012) 112 [arXiv:1203.2160] [INSPIRE].

[45] H. Spiesberger, Inclusive D-meson production at the LHC, arXiv:1205.7000 [INSPIRE].

[46] M. Klasen and M. Brandt, Parton densities from LHC vector boson production at small and large transverse momenta, Phys. Rev. D 88 (2013) 054002 [arXiv:1305.5677] [INSPIRE].

[47] M. Brandt, M. Klasen and F. König, Nuclear parton density modifications from low-mass lepton pair production at the LHC, Nucl. Phys. A 927 (2014) 78 [arXiv:1401.6817] [INSPIRE]. 